\title{
A rare case report of ovarian torsion with sigmoid volvulus
}

\author{
Shraddha A. Mevada*, Archana A. Bhosale, Madhuri A. Mehendale
}

Department of Obstetrics and Gynecology, Lokmanya Tilak Muncipal College and General Hospital, Sion, Mumbai, Maharashtra, India

Received: 29 April 2020

Accepted: 28 May 2020

\section{*Correspondence:}

Dr. Shraddha A. Mevada,

E-mail: drmevadashraddha@gmail.com

Copyright: (C) the author(s), publisher and licensee Medip Academy. This is an open-access article distributed under the terms of the Creative Commons Attribution Non-Commercial License, which permits unrestricted non-commercial use, distribution, and reproduction in any medium, provided the original work is properly cited.

\begin{abstract}
Ovarian torsion results from twisting of the ovary about the suspensory ligament, which contains the ovarian artery and vein, lymphatic's, and nerves. Volvulus is a torsion of a segment of the alimentary tract, that often leads to intestinal obstruction. Ovarian torsion leading to sigmoid volvulus is the rarest complication which authors found in this case. Hence the case was presented. A 28 -year-old women presented with acute pain in abdomen since 14 hours, followed by 2 episodes of vomiting, abdominal distension since 10 hours. Plain X-ray Abdomen erect was done which showed 'Coffee bean' sign with multiple air fluid levels suggestive of sigmoid volvulus. On laparotomy, after opening the peritoneum, large right ovarian cyst around $12 \times 11 \times 10 \mathrm{~cm}$ with solid and haemorrhagic content with long pedicle around $8 \mathrm{~cm}$ with 3 turns of torsion was noted. Abutting the ovarian mass, sigmoid colon was seen twisted around its mesentery including the twisted ovarian pedicle. Hence, the twisted component included the twisted ovarian pedicle and twisted sigmoid mesentery. Stepwise detorsion of ovarian pedicle followed by oophorectomy was done. For sigmoid volvulus, resection of vascular compromised sigmoid colon and descending colon stoma was done. Reanastomosis was done later after 3 months post operatively.
\end{abstract}

Keywords: Abdominal pain, Coffee bean sign, Ovarian torsion, Sigmoid volvulus, Vomiting

\section{INTRODUCTION}

Volvulus is a torsion of a segment of the alimentary tract, that often leads to intestinal obstruction. The most common sites of volvulus are the sigmoid colon and cecum. Anatomic features predisposing to sigmoid volvulus include a redundant sigmoid colon that has a narrow mesenteric attachment, but the exact pathogenesis underlying sigmoid torsion has not been well known.

Most of patients with sigmoid volvulus present with abdominal pain, nausea, abdominal distension, and constipation; vomiting is not common. However, some patients (especially younger patients) will have a more insidious presentation with recurrent attacks of abdominal pain, that resolve presumably due to spontaneous detorsion. ${ }^{1-3}$ Ovarian torsion results from twisting of the ovary about the suspensory ligament, which contains the ovarian artery and vein, lymphatics, and nerves. Although ovarian torsion may occur at any age, it is most common during the reproductive years, with up to $20 \%$ of cases occurring during pregnancy. ${ }^{4}$

Symptoms are nonspecific but often include acute onset of sharp lower abdominal pain and tenderness with possible palpable mass and peritoneal signs. Treatment is surgical.

\section{CASE REPORT}

A 28-year-old women presented with acute pain in abdomen since 14 hours, followed by 2 episodes of vomiting, abdominal distension since 10 hours.

Menstrual history suggested normal menstrual cycles. She was day 10 of menstrual cycle. 
Obstetric history was P1L1, with one male child full term vaginal delivery 2 years of age.

On examination, pulse was 110 beats per minute, blood pressure: $90 / 60 \mathrm{mmHg}$. Respiratory rate was $20 /$ minute.

Per abdomen examination revealed severe guarding, rigidity and tenderness on palpation, distension was present.

Per vaginal examination was done which showed minimal right forniceal tenderness, cervical os parous, no active bleeding.

The patient was evaluated with basic blood investigations which showed complete hemogram with haemoglobin of $9 \mathrm{~g} \%$, total leucocyte count: $20,000 / \mathrm{mm}^{3}$, platelet count: 3.5 lac. liver and renal function test were normal. Coagulation profile was normal.

Plain X-ray abdomen erect was done which showed 'coffee bean' sign with multiple air fluid levels suggestive of Sigmoid volvulus (Figure 1).

Patient was taken up for emergency exploratory laparotomy with proper consent and all pre-requisites.

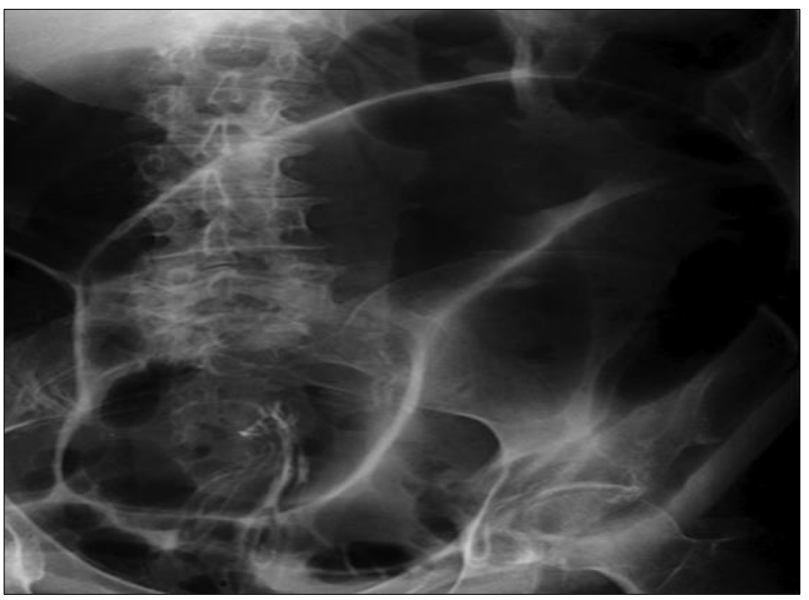

Figure 1: X-ray abdomen with sigmoid volvulus with "coffee bean" appearance.

On laparotomy, after opening the peritoneum, large right ovarian cyst around $12 \times 11 \times 10 \mathrm{~cm}$ with solid and haemorrhagic content with long pedicle around $8 \mathrm{~cm}$ with 3 turns of torsion was noted. Abutting the ovarian mass, sigmoid colon was seen twisted around its mesentery including the twisted ovarian pedicle. Hence, the twisted component included the twisted ovarian pedicle and twisted sigmoid mesentery. Stepwise detorsion of ovarian pedicle followed by oophorectomy was done (Figure 2). For sigmoid volvulus, resection of vascular compromised sigmoid colon and descending colon stoma was done. Specimen was sent for histopathological examination. Reanastomosis was done later after 3months post operatively.

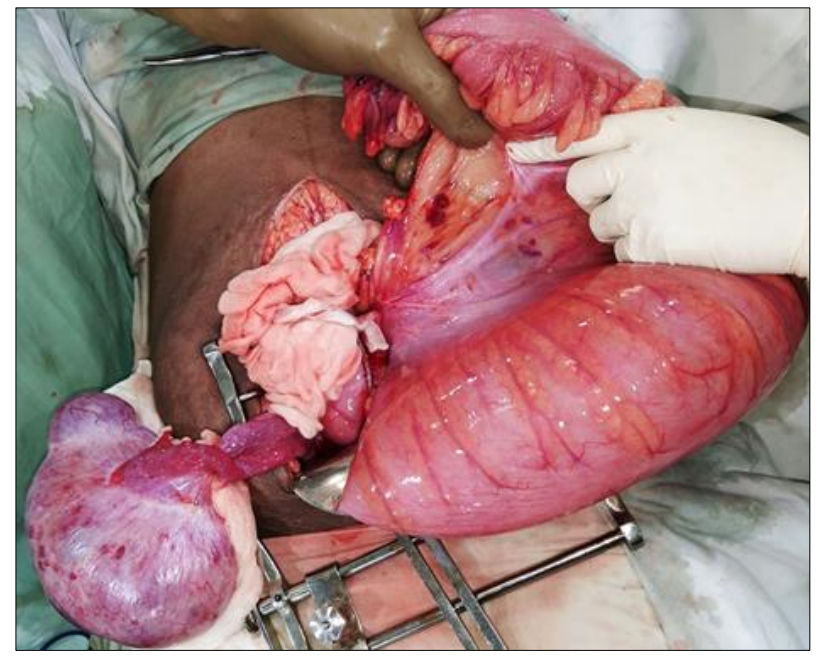

Figure 2: Intraoperative finding of right detorsion of ovarian mass and sigmoid mesentery.

Histopathological report was suggestive of benign serous cystadenoma and sigmoid volvulus with area of necrosis.

\section{DISCUSSION}

Until now, authors have not seen any published article on ovarian torsion and coexistent sigmoid volvulus. A large ovarian cyst might also cause an abnormally elongated sigmoid colon. In this case, it could be the long pedicle of ovarian mass, which must have undergone torsion, with abutted sigmoid colon undergoing volvulus.

Sigmoid volvulus is thought to happen when a loop of the sigmoid colon twists around its mesentery. Obstruction of the intestinal lumen and impairment of vascular perfusion occur when the degree of torsion exceeds 180 and 360 , respectively. ${ }^{5}$ The majority of patients with sigmoid volvulus present similar to the patient presented above with gradual onset of progressive abdominal pain, nausea, distension, and constipation plain abdominal radiographs may help the diagnosis; however, CT, magnetic resonance imaging, and flexible endoscopy are more accurate. ${ }^{6}$ Several radiologic diagnostic signs are described, such as omega or horseshoe sign, bird's beak sign, Y sign, northern exposure sign, coffee bean sign, bent inner tube or ace of spades sign, left pelvic overlap or left flank overlap sign, liver overlap sign, the whirl sign, and empty left iliac fossa sign. Surgeons generally advise a 2-step approach, first an endoscopic derotation followed by a subsequent elective surgical correction by colopexy. Sigmoidoscopy is the initial treatment for those patients without peritoneal signs. Factors that may predispose to sigmoid volvulus include a long sigmoid colon with a narrow mesenteric attachment and colonic motility problems. Constipation may cause elongation and dilatation of the sigmoid colon predisposing patients to sigmoid volvulus. Colonic motility problems may predispose to torsion of the sigmoid colon. ${ }^{3}$ High incidence of sigmoid volvulus has been reported in South America, Africa, and India, which has been attributed to 
the higher-fibre diet. Pregnancy increases the incidence of sigmoid volvulus as well because the enlarging uterus can cause a redundant or abnormally long sigmoid colon.

Funding: No funding sources

Conflict of interest: None declared

Ethical approval: Not required

\section{REFERENCES}

1. Krupsky S, Halevy A, Orda R. Sigmoid volvulus in adolescence. J Clin Gastroenterol. 1987;9:467.

2. Mangiante EC, Croce MA, Fabian TC, Britt LG. Sigmoid volvulus. A four-decade experience. Am Surg. 1989;55:41.

3. Retraction. Clinical presentation and diagnosis of sigmoid volvulus: outcomes of 40-year and 859patient experience. J Gastroenterol Hepatol. 2009;24:1154.
4. Chang HC, Bhatt S, Dogra VS. Pearls and pitfalls in diagnosis of ovarian torsion. Radio Graphics. 2008;28:1355-68.

5. Shepherd JJ. The epidemiology and clinical presentation of sigmoid volvulus. $\mathrm{Br} \mathrm{J}$ Surg. 1969;56:353.

6. Schagen van Leeuwen JH. Sigmoid volvulus in a West African population. Dis Colon Rectum. $1985 ; 28: 712$.

Cite this article as: Mevada SA, Bhosale AA, Mehendale MA. A rare case report of ovarian torsion with sigmoid volvulus. Int J Reprod Contracept Obstet Gynecol 2020;9:3055-7. 